\title{
The Relationship between Acculturation and Experimental Pain Sensitivity in Asian Americans with Knee Osteoarthritis
}

\author{
Hyochol Ahn (D), ${ }^{1}$ Setor K. Sorkpor, ${ }^{1}$ Miyong Kim, ${ }^{2}$ Hongyu Miao, ${ }^{3}$ Chengxue Zhong, ${ }^{3}$ \\ Jing Wang, ${ }^{4}$ Debra Lyon, ${ }^{5}$ and Roger B. Fillingim ${ }^{6}$ \\ ${ }^{1}$ Cizik School of Nursing, University of Texas Health Science Center at Houston, Houston, Texas, USA \\ ${ }^{2}$ School of Nursing, University of Texas, Austin, Texas, USA \\ ${ }^{3}$ School of Public Health, University of Texas Health Science Center at Houston, Houston, Texas, USA \\ ${ }^{4}$ School of Nursing, University of Texas Health Science Center at San Antonio, San Antonio, Texas, USA \\ ${ }^{5}$ College of Nursing, University of Florida, Gainesville, Florida, USA \\ ${ }^{6}$ University of Florida, Pain Research and Intervention Center of Excellence, Gainesville, Florida, USA \\ Correspondence should be addressed to Hyochol Ahn; hyochol.ahn@uth.tmc.edu
}

Received 31 July 2018; Accepted 18 November 2018; Published 29 November 2018

Academic Editor: Massimiliano Valeriani

Copyright $\odot 2018$ Hyochol Ahn et al. This is an open access article distributed under the Creative Commons Attribution License, which permits unrestricted use, distribution, and reproduction in any medium, provided the original work is properly cited.

\begin{abstract}
Multiple studies in healthy populations and clinical samples have shown that ethnic minorities have greater pain sensitivity than their majority counterparts. Acculturation is speculated to be one of the sociocultural factors contributing to pain sensitivity since cultural beliefs and practices can influence the way patients perceive and respond to pain. However, the relationship of acculturation to pain sensitivity in minority populations remains poorly understood. Therefore, in this cross-sectional study, we examined the relationship between acculturation and experimental pain sensitivity in 50 Asian Americans residing in North Central Florida with knee osteoarthritis pain. The Suinn-Lew Asian Self Identity Acculturation Scale was used to assess acculturation, and multimodal quantitative sensory testing was performed to measure experimental sensitivity, including heat pain tolerance, pressure pain threshold, and punctate mechanical pain. Descriptive and regression analyses were performed. Participants' mean age was 55.7 years, and about half of this sample were Korean American (56\%). The participants had lived in the United States for 21 years on average. Regression analyses indicated that lower acculturation to American culture may contribute to greater experimental pain sensitivity. Asian Americans who were more acculturated to the American culture had higher heat pain tolerance (beta $=0.61, P=0.01$ ), higher pressure pain threshold (beta $=0.59, P=0.02$ ), and lower ratings of punctate mechanical pain (beta $=-0.70, P<0.01$ ). These findings add to the literature regarding sociocultural factors associated with pain in Asian Americans; additional research with a larger and more diverse sample of Asian Americans is warranted for crossvalidation.
\end{abstract}

\section{Introduction}

Chronic pain affects 100 million people in the United States and produces annual costs of up to $\$ 635$ billion $[1,2]$. Arthritis is one of the leading causes of pain, impairments of activities in daily life, and disability in people aged 45 and above $[3,4]$. Knee osteoarthritis $(\mathrm{OA})$ is the most common of the arthritic conditions [4-6], and racial/ethnic group differences in knee OA prevalence and its adverse effects have been widely documented [7]. Indeed, some evidence shows that the prevalence of knee OA is higher by $16-75 \%$ in Asian females than age-matched White females [7-9].

Ethnic group disparities in health outcomes are well documented but limited to one or two ethnic minority groups, who have poor outcomes in comparison with Whites. Despite several studies demonstrating ethnic differences in pain $[10,11]$, little research has examined pain experiences in Asians Americans. While it is generally believed that Asian Americans are less sensitive to pain or that their pain experiences do not differ from those of Whites 
[12], recent studies have reported greater experimental pain sensitivity in Asians than in Whites [13-18].

The National Academy of Medicine (previously Institute of Medicine) highlights the importance of research that promotes health equity and eliminates health disparities by investigating the interplay of multidimensional determinants of health and wellness for all populations and that applies this knowledge to the design of personalized interventions [2]. Because the biopsychosocial model of pain hypothesizes that pain is dynamically affected by biological, psychological, and sociocultural factors [19], it is important to understand the sociocultural factors that impact health disparities in pain among Asian Americans. Acculturation, an essential cultural factor that significantly impacts Asian immigrants' health, is defined as the process of cultural adaptation that takes place when an individual has a prolonged exposure to a new culture and is speculated to affect pain sensitivity, since cultural beliefs and practices can influence the way patients perceive and respond to pain $[20,21]$. The literature has indicated that ethnic minority groups with a relatively shorter history of immigration may develop more sensitivity to pain because they must deal with the stresses of acculturation in their new environment $[22,23]$. Although Asian Americans were the fastest growing ethnic group in the United States between 2000 and 2010 [24], the previous studies have not explored the contribution of acculturation to pain sensitivity in this population. Therefore, the aim of this study was to examine the relationship between acculturation and experimental pain sensitivity in Asian Americans with knee OA pain. We hypothesized that lower levels of acculturation to the American culture in Asian Americans may contribute to greater experimental pain sensitivity.

\section{Materials and Methods}

2.1. Participants. Fifty Asian Americans aged 45 to 85 with symptomatic knee OA pain were recruited in North Central Florida via posted fliers and an e-mail advertisement sent to Asian community LISTSERVS. The individuals were provided information about the study, and interested candidates underwent screening for eligibility. Inclusion criteria included Asian Americans who could speak and read English, 45 to 85 years of age, and self-reported knee OA pain. According to American College of Rheumatology criteria [25], participants should meet at least 3 of 6 criteria, including age $>50$ years, stiffness $<30$ minutes, crepitus, bony tenderness, bony enlargement, and no palpable warmth. One of our study team members who is a nationally certified nurse practitioner assessed participants using these inclusion criteria. Exclusion criteria included serious medical illness such as heart failure or a history of acute myocardial infarction, peripheral neuropathy, systemic rheumatic disorders, daily opioid use, cognitive impairment, or hospitalization within the preceding year for psychiatric illness. All procedures were approved by the institutional review board at the University of Florida before commencement, and all patients gave oral and written informed consent prior to participation.

\subsection{Measures}

2.2.1. Acculturation. The Suinn-Lew Asian Self Identity Acculturation (SL-ASIA) Scale in English [26] was used to measure acculturation in Asian Americans. This scale consists of 21 items that measure the degree of acculturation in friendship, language, behaviors, and diet. Each item on the scale is rated from 1 to 5; higher scores indicate greater acculturation to American culture. The 21 items were averaged to generate final acculturation scores that reflected the overall level of acculturation. The SL-ASIA has been reported to have good internal consistency (Cronbach's $\alpha=$ $0.88-0.90)$ and has been used widely in research involving Asian Americans with a wide range of ages, including older adults [27-29].

2.2.2. Experimental Pain Sensitivity. Heat pain tolerance was measured on both the index knee and the ipsilateral ventral forearm with contact heat stimuli delivered via a Medoc Pathway Neurosensory Analyzer (Ramat Yishai, Israel) using an ascending method of limits. The temperature began at $32^{\circ} \mathrm{C}$ and increased at a rate of $0.5^{\circ} \mathrm{C}$ per second until participants responded by pressing a button on a handheld device when they "no longer feel able to tolerate the pain." Heat was applied to each of the two locations three times, and the temperatures of the three individual trials at each body site were averaged to generate heat pain tolerance at each site. We assessed heat pain tolerance, because tolerance is thought to reflect the affective-motivational dimension.

Pressure pain thresholds were measured by a handheld Medoc digital pressure algometer (Algomed) at a constant rate of $30 \mathrm{kPa}$ per second at 5 sites: the medial aspect of the index knee, lateral aspect of the index knee, ipsilateral quadriceps, trapezius, and epicondyle. The order of testing sites was randomized and counterbalanced. To assess pressure pain thresholds, participants were instructed to press the button when the sensation "first becomes painful." Pressure was applied to each of the five locations three times, and the results of the three individual trials at each body site were averaged to generate pressure pain threshold at each site.

Punctate pain sensitivity was measured using a calibrated nylon monofilament delivering a target force of $300 \mathrm{~g}$ applied on the index patella as well as the back of the ipsilateral hand. Participants provided numerical pain intensity ratings on a scale from 0 (no pain sensation) to 100 (the most intense pain sensation imaginable) after 10 contacts at a rate of 1 contact per second at each of the two locations. This procedure was performed twice at each site, and the results of the two individual trials at each body site were averaged to generate punctate mechanical pain at each site.

2.3. Statistical Analysis. All analyses were conducted with SAS version 9.4. Descriptive statistics appropriate for the level of measurement were used to validate values and evaluate missing and variable distributions. For purposes of 
variable reduction, we created composite measures of pain sensitivity. First, the normality of data distribution was checked by using the Shapiro-Wilk test for the four pain measurements. Only the normality of pressure pain threshold did not hold; therefore, the Box-Cox transformation was applied on raw data of pressure pain threshold. After the Box-Cox transformation on pressure pain threshold, $z$-scores were computed for heat pain tolerance measurements at the arm and knee; pressure pain threshold measures at the medial knee, lateral knee, quadriceps, trapezius, and epicondyle; and punctate pain measurements at the patella and hand. Then, the $z$-scores for each pain measure were averaged across body sites to derive an overall measure of heat pain tolerance, pressure pain threshold, and punctate pain. We created $z$-scores for each pain modality for variable reduction, as in prior research. Specifically, previous factor analytic studies have demonstrated that laboratory pain measures typically aggregate within pain modality. That is, factor analytic solutions generally reveal a heat pain factor, a pressure pain factor, and so on [30-32]. Thus, investigators have commonly computed pain index scores by averaging $z$-scores within pain modalities $[33,34]$. This allows variable reduction, while also placing all pain measures on a common metric without altering the distributional characteristics of the underlying data. These $z$-scores were used as outcomes to analyze the relationship between pain sensitivity and acculturation. Gender, age, acculturation, and BMI were used to fit separate linear regression models for each experimental pain measure. SAS PROC GLM was employed to estimate the parameters after fixing the age, BMI, and gender in our model.

\section{Results}

3.1. Demographic Characteristics. Fifty Asian Americans were enrolled for this study (see Table 1 for details). About half of this sample was Korean Americans (56\%), mean age was 55.66 (standard deviation $(\mathrm{SD})=7.81$ ), and the participants had lived in the United States for about 21 years $(\mathrm{SD}=15.15)$. The mean BMI in the sample was $24.18(\mathrm{SD}=$ 3.03). Participants had an average SL-ASIA score of 2.18 (SD $=0.54$ ), indicating an average of low acculturation to the American culture in the study sample.

\subsection{Relationship between Acculturation and Experimental} Pain Sensitivity. There was a signification relationship between acculturation and all three pain sensitivity measures (Table 2). Asian Americans who were more acculturated to the American culture had higher heat pain tolerance (beta $=0.61, P=0.01$ ), higher pressure pain threshold (beta $=0.59, P=0.02$ ), and lower rating of punctate mechanical pain (beta $=-0.70, P<0.01$ ). In addition, male Asian Americans in the United States had greater heat pain tolerance (beta $=0.87, P<0.01$ ), greater pressure pain threshold (beta $=0.79, P<0.01$ ), and lower rating of punctate mechanical pain (beta $=-0.52, P=0.05$ ) than did female Asian Americans.
TABle 1: Demographic and clinical characteristics of participants.

\begin{tabular}{lc}
\hline Characteristics & Total \\
\hline Age $($ year$)$, mean $(\mathrm{SD})$ & $55.66(7.81)$ \\
$\mathrm{BMI},\left(\mathrm{kg} / \mathrm{m}^{2}\right)$, mean $(\mathrm{SD})$ & $24.18(3.03)$ \\
Gender, $n(\%)$ & \\
$\quad$ Male & $19(38)$ \\
Female & $31(62)$ \\
Original country, $n(\%)$ & \\
Korea & $28(56)$ \\
China & $9(18)$ \\
Japan & $7(14)$ \\
Filipino & $5(10)$ \\
India & $1(2)$ \\
Length of stay at the USA (year), mean $(\mathrm{SD})$ & $21.38(15.15)$ \\
Education, $n(\%)$ & \\
Less than high school & $1(2)$ \\
High school & $7(14)$ \\
Some college & $8(16)$ \\
Bachelor's degree & $14(28)$ \\
Graduate degree & $20(40)$ \\
Heat pain tolerance $\left({ }^{\circ} \mathrm{C}\right)$, mean $(\mathrm{SD})^{\dagger}$ & $41.84(3.92)$ \\
Pressure pain threshold $(\mathrm{kPa})$, mean $(\mathrm{SD})^{\dagger}$ & $181.23(85.01)$ \\
Punctate pain, mean $(\mathrm{SD})^{\dagger}$ & $40.43(23.69)$ \\
SL-AISA, mean $(\mathrm{SD})$ & $2.18(0.55)$ \\
\hline
\end{tabular}

Note. BMI, body mass index. Data are presented as mean (standard deviation) or number (percentage). Measured on the SL-ASIA, total scores range from 1 to 5 , with higher scores indicating more acculturation to American culture. ${ }^{\dagger}$ Overall average score.

TABLE 2: Results of regression model.

\begin{tabular}{|c|c|c|c|c|}
\hline Variable & Beta & Standard error & $t$ value & $P$ value \\
\hline \multicolumn{5}{|c|}{ Heat pain tolerance* } \\
\hline Acculturation & 0.61 & 0.23 & 2.69 & 0.01 \\
\hline Age & -0.01 & 0.02 & -0.70 & 0.49 \\
\hline Male & 0.87 & 0.25 & 3.45 & $<0.01$ \\
\hline BMI & 0.03 & 0.04 & 0.84 & 0.40 \\
\hline \multicolumn{5}{|c|}{ Pressure pain threshold* } \\
\hline Acculturation & 0.59 & 0.24 & 2.48 & 0.02 \\
\hline Age & 0.01 & 0.02 & 0.82 & 0.41 \\
\hline Male & 0.79 & 0.27 & 2.96 & $<0.01$ \\
\hline BMI & 0.05 & 0.04 & 1.16 & 0.25 \\
\hline \multicolumn{5}{|l|}{ Punctate pain* } \\
\hline Acculturation & -0.70 & 0.23 & -2.99 & $<0.01$ \\
\hline Age & 0.01 & 0.02 & 0.70 & 0.49 \\
\hline Male & -0.52 & 0.26 & -1.97 & 0.05 \\
\hline BMI & -0.02 & 0.04 & -0.38 & 0.71 \\
\hline
\end{tabular}

Note. BMI, body mass index. R-square: heat pain tolerance $=0.32$, pressure pain threshold $=0.25$, punctate pain $=0.22 .{ }^{*}$ Average $z$-score.

\section{Discussion}

In this study to identify the relationship between acculturation and experimental pain sensitivity among Asian Americans, the fastest growing ethnic group in the United States [6], we found that participants with higher acculturation to American culture had lower experimental pain sensitivity (i.e., higher heat pain tolerance, higher pressure pain threshold, and lower punctate pain). We also found no statistically significant interaction between acculturation 
and gender: male participants reported a higher tolerance and pain threshold than did female participants, regardless of acculturation.

The findings of the present study are consistent with previous evidence showing that individuals with low acculturation to their host culture experience heightened pain. For example, Edrington et al. [35] reported that lower acculturation to American culture was associated with higher pain intensity and pain interference among Asian Americans with cancer. Similarly, Chan et al. [36] reported that first-generation Asian Americans showed greater cold pain sensitivity than European Americans, while secondgeneration Asian Americans did not differ from the European American group. Also, Palmer et al. [37] found a negative association between acculturation to British culture and prevalence of widespread pain among Asians in the United Kingdom.

The exact mechanisms underlying the relationship between acculturation and pain are unclear, but one possible explanation for the amplified pain sensitivity in relation to low acculturation may be the result of stress arising from conflict between the heritage culture and host culture $[17,38]$. These negative experiences may diminish one's sense of self-control, which in turn may lead to higher pain sensitivity. Another possibility is that lower acculturation may increase discriminatory treatment of minorities, which represents a potentially potent chronic stress exposure. Also, lower acculturation could be associated with greater disparities in provision of healthcare [39]. Hence, Asian Americans with lower acculturation may have less familiarization with the language, food, customs, social norms, and values of American culture, which likely produces crosscultural stresses and thereby increases sensitivity to pain.

This study's results should be interpreted in light of several limitations. First, the sample of 50 Asian Americans originated from five different countries, with about half from Korea. Our sample, therefore, was not truly representative of the Asian American population. Second, Asian Americans in our study were limited to those who could speak and read English, which might have excluded people in the extremely low acculturation spectrum and therefore imposed some interference challenges of findings. It is reasonable to speculate that the magnitude of relationship between acculturation and pain sensitivity might be even greater in non-English-speaking individuals in the United States. Third, we did not consider all confounding variables, but we did statistically control for age, gender, and BMI. Given the small sample size, confounding variables were restricted to pain-related individual factors. Fourth, the data collection was based on a cross-sectional study design, consequently limiting our ability to draw causal inferences.

Future studies conducted with a larger and more heterogeneous sample are needed to validate these findings. Nonetheless, the following clinical implications warrant consideration. Healthcare providers should proactively assess multiple dimensions of pain, including cultural aspects, in Asian American patients. In addition, there is a significant need for culturally sensitive pain interventions to improve pain management in this vulnerable population.
The findings of the present study lay the groundwork for further research to investigate the mechanisms by which acculturation influences pain sensitivity. First, future studies with larger samples could address geographic location of original home country, because there may be subgroup differences among Asian Americans. A more heterogeneous sample would provide a better representative sample allowing for between-group estimations of differences in pain intensity rating in relation to acculturation. Second, future studies are needed to investigate cultural beliefs and values that reduce or heighten pain sensitivity. Finally, future studies that incorporate additional biological and psychological measures are needed to elucidate underlying mechanisms.

\section{Conclusion}

The relationship between acculturation and experimental pain sensitivity in older adults with chronic pain has rarely been examined. The results from our study suggest that lower acculturation to American culture may be associated with heightened pain sensitivity among ethnic minorities in the United States. The present study adds to the growing literature that acculturation has an influence on pain sensitivity. Further investigation is needed to determine the role of specific cultural factors in heightened pain and to ensure that ethnic group disparities in pain are ameliorated via programs directed at promoting acculturation among Asian Americans.

\section{Data Availability}

The data used to support the findings of this study are available from the corresponding author upon request.

\section{Disclosure}

Components of this work were presented as posters at the 9th Congress of the European Pain Federation, Vienna, Austria.

\section{Conflicts of Interest}

All authors report no conflicts of interests.

\section{Acknowledgments}

This project was supported in part by the NIH/NCATS Clinical and Translational Science Award to the University of Florida UL1 TR000064 and by the NIH/NIA grant R37AG033906. Editorial support with manuscript development was provided by the Cain Center for Nursing Research and the Center for Transdisciplinary Collaborative Research in Self-management Science (P30, NR015335) at the University of Texas at Austin School of Nursing.

\section{References}

[1] D. J. Gaskin and P. Richard, "The economic costs of pain in the United States," Journal of Pain, vol. 13, no. 8, pp. 715-724, 2012. 
[2] Institute of Medicine, Relieving Pain in American: A Blueprint for Transforming Prevention, Care, Education, and Research, National Acadmies Press, Washington, DC, USA, 2011.

[3] D. J. Hunter, J. J. McDougall, and F. J. Keefe, "The symptoms of osteoarthritis and the genesis of pain," Rheumatic Disease Clinics of North America, vol. 34, no. 3, pp. 623-643, 2008.

[4] R. C. Lawrence, D. T. Felson, C. G. Helmick et al., "Estimates of the prevalence of arthritis and other rheumatic conditions in the United States: part II," Arthritis \& Rheumatism, vol. 58, no. 1, pp. 26-35, 2008.

[5] D. T. Felson, R. C. Lawrence, P. A. Dieppe et al., "Osteoarthritis: new insights. Part 1: the disease and its risk factors," Annals of Internal Medicine, vol. 133, no. 8, pp. 635-646, 2000.

[6] J. M. Jordan, C. G. Helmick, J. B. Renner et al., "Prevalence of knee symptoms and radiographic and symptomatic knee osteoarthritis in african Americans and Caucasians: the Johnston County osteoarthritis project," Journal of Rheumatology, vol. 34, no. 1, pp. 172-180, 2007.

[7] K. D. Allen, "Racial and ethnic disparities in osteoarthritis phenotypes," Current Opinion in Rheumatology, vol. 22, no. 5, pp. 528-532, 2010.

[8] Y. Zhang, L. Xu, M. C. Nevitt et al., "Comparison of the prevalence of knee osteoarthritis between the elderly Chinese population in Beijing and whites in the United States: the Beijing osteoarthritis study," Arthritis \& Rheumatism, vol. 44, no. 9, pp. 2065-2071, 2001.

[9] D. T. Felson, M. C. Nevitt, Y. Zhang et al., "High prevalence of lateral knee osteoarthritis in Beijing Chinese compared with Framingham Caucasian subjects," Arthritis \& Rheumatism, vol. 46, no. 5, pp. 1217-1222, 2002.

[10] A. Cintron and R. S. Morrison, "Pain and ethnicity in the United States: a systematic review," Journal of Palliative Medicine, vol. 9, no. 6, pp. 1454-1473, 2006.

[11] C. R. Green, T. A. Baker, E. M. Smith, and Y. Sato, "The effect of race in older adults presenting for chronic pain management: a comparative study of black and white Americans," Journal of Pain, vol. 4, no. 2, pp. 82-90, 2003.

[12] E. O. Im, W. Chee, E. Guevara et al., "Gender and ethnic differences in cancer pain experience: a multiethnic survey in the United States," Nursing Research, vol. 56, no. 5, pp. 296-306, 2007.

[13] Q. Lu, L. Zeltzer, and J. Tsao, "Multiethnic differences in responses to laboratory pain stimuli among children," Health Psychology, vol. 32, no. 8, pp. 905-914, 2013.

[14] L. N. Rowell, B. Mechlinl, E. Jil, M. Addamol, and S. S. Girdlerl, "Asians differ from non-hispanic whites in experimental pain sensitivity," European Journal of Pain, vol. 15, no. 7, pp. 764-771, 2011.

[15] A. Y. Hsieh, D. A. Tripp, L.-J. Ji, and M. J. L. Sullivan, "Comparisons of catastrophizing, pain attitudes, and coldpressor pain experience between Chinese and European Canadian young adults," Journal of Pain, vol. 11, no. 11, pp. 1187-1194, 2010.

[16] O. Komiyama, K. Wang, P. Svensson, L. Arendt-Nielsen, M. Kawara, and A. De Laat, "Ethnic differences regarding sensory, pain, and reflex responses in the trigeminal region," Clinical Neurophysiology, vol. 120, no. 2, pp. 384389, 2009.

[17] H. Ahn, M. Weaver, D. Lyon, E. Choi, and R. B. Fillingim, "Depression and pain in asian and white Americans with knee osteoarthritis," Journal of Pain, vol. 18, no. 10, pp. 1229-1236, 2017.

[18] H. Ahn, M. Weaver, D. E. Lyon et al., "Differences in clinical pain and experimental pain sensitivity between asian
Americans and whites with knee osteoarthritis," Clinical Journal of Pain, vol. 33, no. 2, pp. 174-180, 2017.

[19] R. B. Fillingim, "Individual differences in pain responses," Current Rheumatology Reports, vol. 7, no. 5, pp. 342-347, 2005.

[20] N. Henschke, E. Lorenz, R. Pokora, Z. A. Michaleff, J. N. A. Quartey, and V. C. Oliveira, "Understanding cultural influences on back pain and back pain research," Best Practice \& Research Clinical Rheumatology, vol. 30, no. 6, pp. 10371049, 2016.

[21] W. S. Shaw, P. Campbell, C. C. Nelson, C. J. Main, and S. J. Linton, "Effects of workplace, family and cultural influences on low back pain: what opportunities exist to address social factors in general consultations?," Best Practice \& Research Clinical Rheumatology, vol. 27, no. 5, pp. 637-648, 2013.

[22] A. C. Mui and S. Y. Kang, "Acculturation stress and depression among Asian immigrant elders," Social Work, vol. 51, no. 3, pp. 243-255, 2006.

[23] C. B. Young, D. Z. Fang, and S. Zisook, "Depression in Asian-American and Caucasian undergraduate students," Journal of Affective Disorders, vol. 125, no. 1-3, pp. 379-382, 2010.

[24] United States Census Bureau, The Asian Population: 2010 Census Briefs, United States Census Bureau, Suitland, MD, USA, 2011, https://www.census.gov/prod/cen2010/briefs/ c2010br-11.pdf.

[25] R. Altman, E. Asch, D. Bloch et al., "Development of criteria for the classification and reporting of osteoarthritis: classification of osteoarthritis of the knee. Diagnostic and therapeutic criteria Committee of the American Rheumatism Association," Arthritis \& Rheumatism, vol. 29, no. 8, pp. 1039-1049, 1986.

[26] S. A. Khan, R. T. Jackson, and B. Momen, "The relationship between diet quality and acculturation of immigrated South asian american adults and their association with metabolic syndrome," PLoS One, vol. 11, no. 6, Article ID e0156851, 2016.

[27] J. W. Ayers, C. R. Hofstetter, P. Usita, V. L. Irvin, S. Kang, and M. F. Hovell, "Sorting out the competing effects of acculturation, immigrant stress, and social support on depression: a report on Korean women in California," Journal of Nervous and Mental Disease, vol. 197, no. 10, pp. 742-747, 2009.

[28] C. R. Hofstetter, M. Hovell, K.-R. Jung, R. Raman, V. Irvin, and $\mathrm{R}$. Ni, "The first puff: forces in smoking initiation among Californians of Korean descent," Nicotine \& Tobacco Research, vol. 9, no. 12, pp. 1277-1286, 2007.

[29] C. R. Hofstetter, M. Hovell, and J. Lee, "Tobacco use and acculturation among Californians of Korean descent: a behavioral epidemiological analysis," Nicotine \& Tobacco Research, vol. 6, no. 3, pp. 481-489, 2004.

[30] B. A. Hastie, J. L. Riley, M. E. Robinson et al., "Cluster analysis of multiple experimental pain modalities," Pain, vol. 116, no. 3, pp. 227-237, 2005.

[31] Y. Cruz-Almeida, J. L. Riley 3rd., and R. B. Fillingim, "Experimental pain phenotype profiles in a racially and ethnically diverse sample of healthy adults," Pain Medicine, vol. 14, no. 11, pp. 1708-1718, 2013.

[32] J. S. Cardoso, J. L. Riley, T. Glover et al., "Experimental pain phenotyping in community-dwelling individuals with knee osteoarthritis," Pain, vol. 157, no. 9, pp. 2104-2114, 2016.

[33] B. A. Hastie, J. L. Riley, L. Kaplan et al., "Ethnicity interacts with the OPRM1 gene in experimental pain sensitivity," Pain, vol. 153, no. 8, pp. 1610-1619, 2012. 
[34] Y. Cruz-Almeida and R. B. Fillingim, "Can quantitative sensory testing move us closer to mechanism-based pain management?," Pain Medicine, vol. 15, no. 1, pp. 61-72, 2014.

[35] J. Edrington, A. Sun, C. Wong et al., "A pilot study of relationships among pain characteristics, mood disturbances, and acculturation in a community sample of Chinese American patients with cancer," Oncology Nursing Forum, vol. 37, no. 2, pp. 172-181, 2010.

[36] M. Y. Chan, T. Hamamura, and K. Janschewitz, "Ethnic differences in physical pain sensitivity: role of acculturation," Pain, vol. 154, no. 1, pp. 119-123, 2013.

[37] B. Palmer, G. Macfarlane, C. Afzal, A. Esmail, A. Silman, and M. Lunt, "Acculturation and the prevalence of pain amongst South Asian minority ethnic groups in the UK," Rheumatology (Oxford), vol. 46, no. 6, pp. 1009-1014, 2007.

[38] Y. J. Cho, Y. Jang, J. Eun Ko, S. H. Lee, and S. K. Moon, "Acculturation, acculturative stress, and depressive symptoms in international migrants: a study with Vietnamese women in South Korea," Journal of Immigrant and Minority Health, vol. 20, no. 5, pp. 1103-1108, 2017.

[39] C. L. Edwards, R. B. Fillingim, and F. Keefe, "Race, ethnicity and pain," Pain, vol. 94, no. 2, pp. 133-137, 2001. 


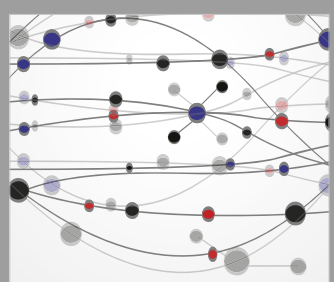

The Scientific World Journal
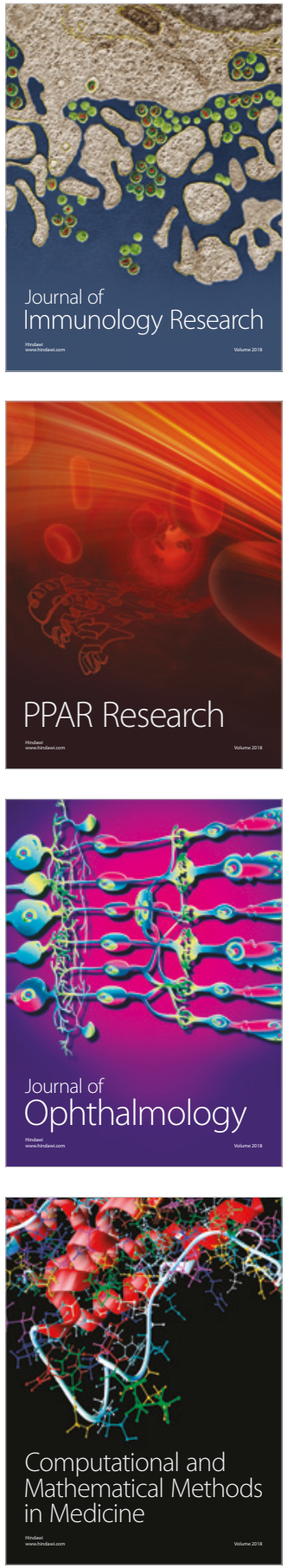

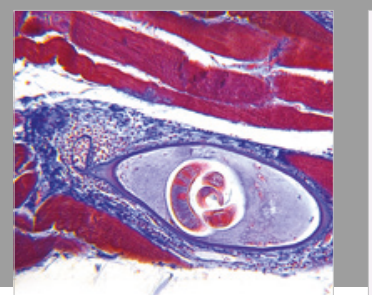

Gastroenterology Research and Practice

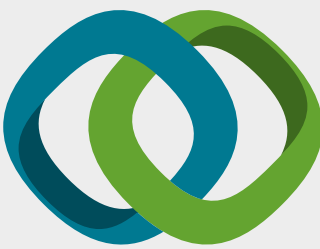

\section{Hindawi}

Submit your manuscripts at

www.hindawi.com
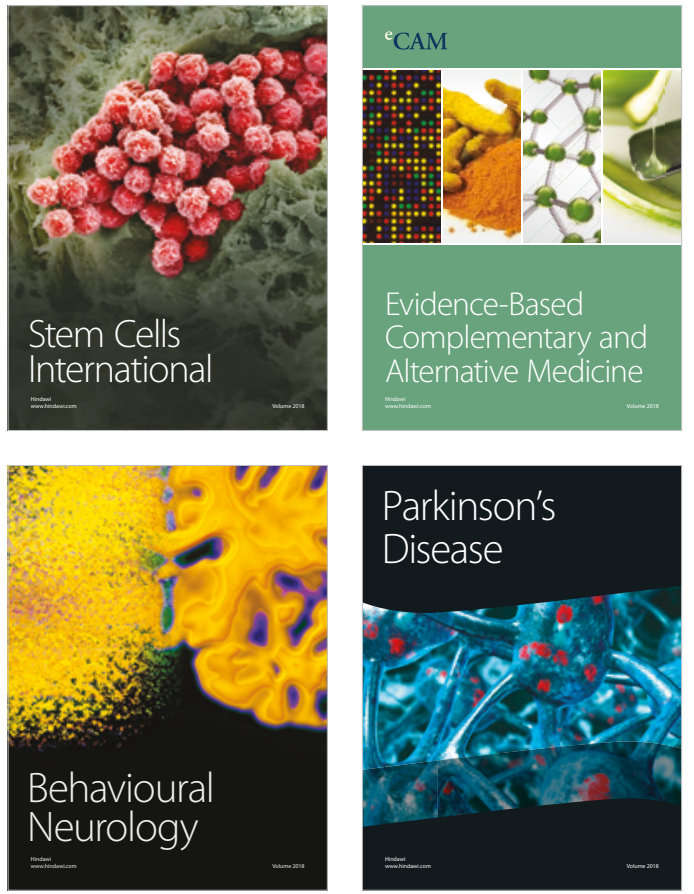

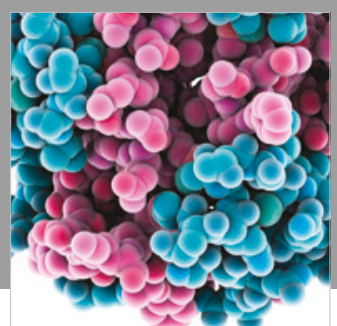

ournal of

Diabetes Research

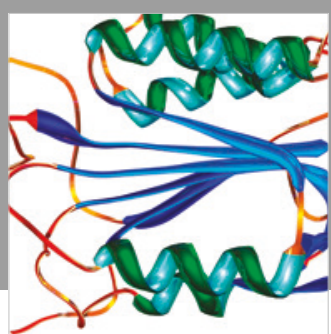

Disease Markers
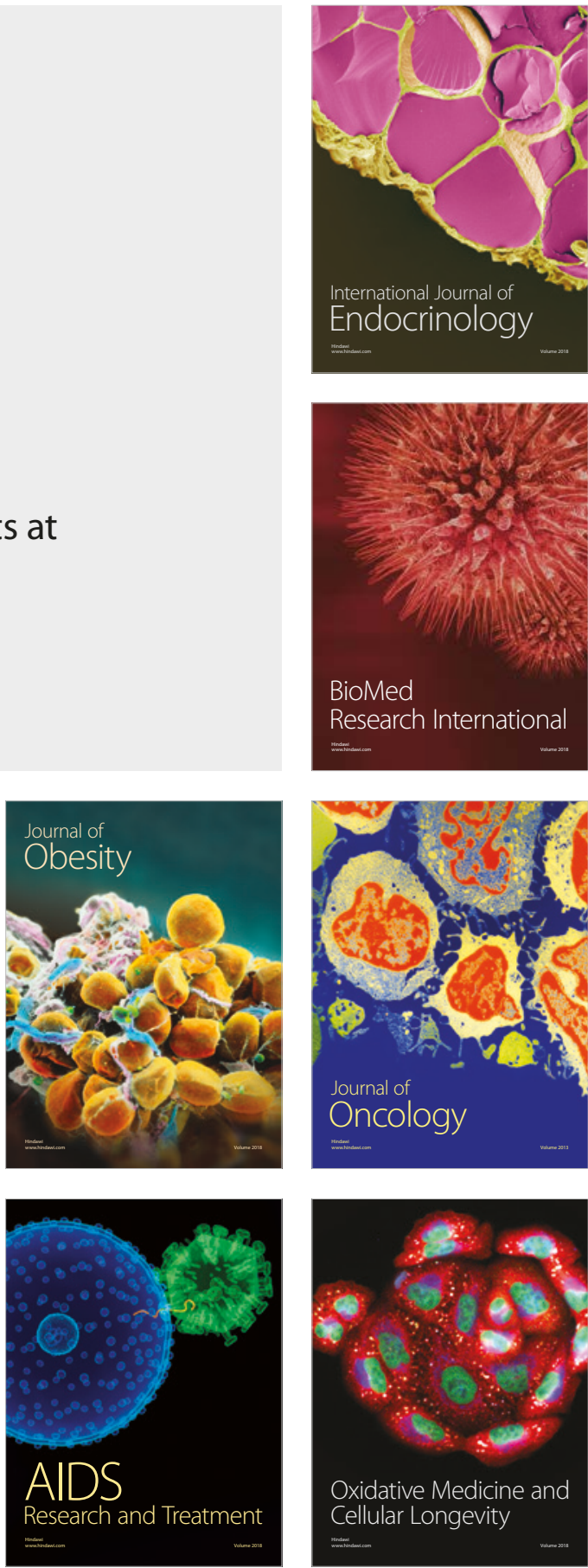